Pacific Journal of Mathematics

UNITARY DILATION FOR COMMUTING CONTRACTIONS 


\title{
UNITARY DILATIONS FOR COMMUTING CONTRACTIONS
}

\author{
STEPHen PARRotT
}

Let $S_{1}, S_{2}, \cdots, S_{n}$ be a set of commuting contraction operators on a Hilbert space $H$, let $U_{1}, U_{2}, \cdots, U_{n}$ be a set of commuting unitary operators on a Hilbert space $K$ containing $H$, and let $P$ be the projection from $K$ to $H$. The set $U_{1}, \cdots, U_{n}$ is called a set of commuting unitary dilations for $S_{1}, \cdots, S_{n}$ provided that

$$
P U_{1}^{m}{ }_{1} U_{2}^{m}{ }_{2} \cdots U_{n}^{m}{ }_{n} x=S_{1}^{m}{ }_{1} S_{2}^{m}{ }_{2} \cdots S_{n}^{m}{ }_{n} x
$$

for all $x$ in $H$ and for all nonnegative integers $m_{1}, m_{2}, \cdots, m_{n}$. Sz.-Nagy proved that a single contraction has a unitary dilation, and Ando showed that any two commuting contractions possess a pair of commuting unitary dilations. This note presents several counterexamples which disprove the corresponding conjecture for three or more contractions.

In $\S 3$, three commuting contractions, $R, S, T$ are constructed which do not have commuting unitary dilations. The operators $R$ and $S$ each have norm one, while the operator $T$ may be chosen to have any norm between zero and one. However, the proof yielding the counterexample fails completely if the operators $R, S, T$ are replaced by $\lambda R, \lambda S, T$ with $0<\lambda<1$, and this raises another question.

It is known that a finite or infinite set of commuting contractions $S_{1}, S_{2}, \cdots$ which satisfies $\sum\left\|S_{k}\right\|^{2} \leqq 1$ possesses a set of commuting unitary dilations. Thus it appears that the "size" of a set of contractions may be relevant to the existence of commuting unitary dilations; and since two of the contractions in $\S 3$ have norm one, it is conceivable that this example might be only a peculiar "boundary" phenomenon. In $\S 4$ this notion is dispelled by a more complicated example showing that three commuting contractions, each of norm strictly less than one, can fail to have commuting unitary dilations. Although the example of $\S 4$ is in most (but not all) respects more powerful than that of $\S 3$, the latter is presented separately because of its simplicity.

Section 3 also observes that a recent result of Sz.-Nagy and Foias is equivalent to Ando's theorem. Section 5 shows that the counterexamples constructed in this paper to the unitary dilation conjecture cannot be used as counterexamples to another well-known conjecture concerning spectral sets.

2. Notation and preliminaries. If $H$ is a subspace of a Hilbert space $K$, the orthogonal projection from $K$ to $H$ will be written as 
$P_{H}$, and the restriction of an operator $S$ to $H$ will be written as $S \mid H$. A contraction operator $S$ on a Hilbert space is a linear operator with $\|S\| \leqq 1$; a proper contraction satisfies $\|S\|<1$.

We shall require a well-known result of Sz.-Nagy which states that the minimal unitary dilation of a contraction is unique up to unitary equivalence.

THEOREM (Sz.-Nagy). Let $S$ be a contraction operator on a Hilbert space $H$, and let $U$ and $U^{\prime}$ be unitary dilations of $S$ to Hilbert spaces $K$ and $K^{\prime}$, respectively, containing $H$. Let $K_{0}$ (resp. $\left.K_{0}^{\prime}\right)$ be the smallest subspace of $K$ (resp. $K^{\prime}$ ) which contains $H$ and reduces $U$ (resp. $\left.U^{\prime}\right)$. Then there is a unitary operator $W$ from $K_{0}$ onto $K_{0}^{\prime}$ such that $W \mid H$ is the identity operator, and $W\left(U \mid K_{0}\right) W^{-1}=$ $U^{\prime} \mid K_{0}^{\prime}$.

The operator $U \mid K_{0}$ is called the minimal unitary dilation for the operator $S$.

3. A simple example. In this section we present a very simple example of three commuting contraction operators which do not possess commuting unitary dilations. Let $H_{0}$ be a Hilbert space of dimension at least two, and let $H=H_{0} \oplus H_{0}$. Let $V$ be any unitary operator on $H_{0}$ which is not a scalar multiple of the identity operator $I$, and let $A$ be any contraction on $H_{0}$ which does not commute with $V$. Define operators $R, S, T$ on $H$ by the operator matrices:

$$
R=\left[\begin{array}{ll}
0 & 0 \\
I & 0
\end{array}\right], S=\left[\begin{array}{ll}
0 & 0 \\
V & 0
\end{array}\right], T=\left[\begin{array}{ll}
0 & 0 \\
A & 0
\end{array}\right] \text {. }
$$

Notice that $R, S, T$ commute no matter how $V$ and $A$ are chosen; in fact, the product of any two of them is 0 . We shall show that these operators cannot have commuting unitary dilations.

The proof is more natural when expressed in functional, rather than sequential, notation and for this reason we introduce the Hilbert space $K_{0}$ of all Fourier series $\sum_{n=-\infty}^{\infty} z^{n} x_{n}$, with the Fourier coefficients $x_{n}$ in $H_{0}$, and $\sum\left\|x_{n}\right\|^{2}<\infty$. The inner product $\langle\cdot, \cdot\rangle$ on $K_{0}$ is defined, as usual, by $\left\langle\sum z^{n} x_{n}, \sum z^{n} y_{n}\right\rangle=\sum\left(x_{n}, y_{n}\right)$, where $(\cdot, \cdot)$ is the inner product on $H_{0}$. Such a Fourier series may be considered to define an honest square-integrable function from the unit circle into $H_{0}$ (see [8] for details), or it may be considered as merely a convenient way of keeping track of the components of a conventional infinite sequence of elements of $H_{0}$. For our purposes it makes no difference.

Let $U$ be the operator on $K_{0}$ defined by: $U\left(\sum z^{n} x_{n}\right)=\sum z^{n+1} x_{n}$. When expressed in sequential notation, $U$ is just the familiar bilateral 
shift acting on a direct sum of copies of $H_{0}$. Further, if $H=H_{0} \oplus H_{0}$ is identified in the obvious way with the subspace of $K_{0}$ consisting of all functions of the form $x_{0}+z x_{1}$, with $x_{0}, x_{1}$ in $H_{0}$, then it is very easy to check that $U$ is the minimal unitary dilation of the operator $R$ defined above.

Now suppose $R, S, T$ have commuting unitary dilations $U_{R}, U_{S}, U_{T}$ acting on a Hilbert space $K$ containing $H$, and let $K_{0}^{\prime}$ be the smallest subspace of $K$ which contains $H$ and reduces $U_{R}$. Then the theorem of Sz.-Nagy quoted in $\S 2$ shows that we may identify $K_{0}^{\prime}$ with the space $K_{0}$ of Fourier series in such a way that $H$ is identified with $\left\{x_{0}+z x_{1} \mid x_{0}, x_{1} \in H_{0}\right\}$ and $U_{R} \mid K_{0}=U$.

Given $x$ in $H$, let $\hat{x}$ be the Fourier series $\sum z^{n} x_{n}$ with $x_{0}=x$ and $x_{n}=0$ for $n \neq 0$. Since $P_{H} U_{S} \mid H=S, U_{S} \hat{x}=w+\sum z^{n} y_{n}$ with $y_{0}=0$, $y_{1}=V x$, and $w$ orthogonal to $K_{0}$. Then, $\|x\|^{2}=\left\|U_{S} \widehat{x}\right\|^{2}=\|V x\|^{2}+$ $\|w\|^{2}+\sum_{n \neq 1}\left\|y_{n}\right\|^{2}$, and since $V$ is an isometry, we must have $y_{n}=0$ for $n \neq 1$ and $w=0$. Since $U_{S}$ commutes with $U_{R}, U_{S}\left(z^{n} x\right)=$ $U_{S}\left(U_{R}^{n} \hat{x}\right)=U_{R}^{n} U_{S} \hat{x}=z^{n+1} V x$. Thus $U_{S}$ maps $K_{0}$ onto itself (which implies that $K_{0}$ reduces $U_{S}$ because $U_{S}$ is unitary), and $U_{S}$ is uniquely determined on $K_{0}$ by the equation above. In fact, $U_{S} \mid K_{0}$ may be considered as multiplication by the operator-valued function $z \rightarrow z V$.

Now let $E$ be the projection on $K_{0}$, and let $\widetilde{T}=E U_{T} \mid K_{0}$. Then, since $K_{0}$ reduces both $U_{R}$ and $U_{S}, E$ commutes with $U_{R}$ and $U_{S}$, and hence $\widetilde{T}$ commutes with both $U=U_{R} \mid K_{0}$ and $U_{S} \mid K_{0}$.

Let $\widetilde{T} \hat{x}=\sum z^{n} L_{n} x$, where this defines $L_{n}$ as operators from $H_{0}$ to $H_{0}$. Since $\widetilde{T}$ commutes with $U$, it is easy to see that $\widetilde{T}$ acts as multiplication by the operator-valued function $z \rightarrow \sum z^{n} L_{n}$, and it is obvious that this will commute with multiplication by $z \rightarrow z V$ if and only if each operator $L_{n}$ commutes with $V$.

More explicitly, we compute: $\widetilde{T} U_{s} \widehat{x}=\widetilde{T}(z V x)=\widetilde{T} U(\widehat{V x})=U \widetilde{T}(\widehat{V x})=$ $\sum z^{n+1} L_{n} V x$ and $U_{S} \widetilde{T} \hat{x}=U_{S}\left(\sum z^{n} L_{n} x\right)=\sum z^{n+1} V L_{n} x$.

But finally we notice that $P_{H} \widetilde{T}\left|H=P_{H} E U_{T}\right| H=P_{H} U_{T} \mid H=T$, and hence $L_{0}=0$ and $L_{1}=A$. Thus if $A$ is chosen to be any contraction which does not commute with $V$, we have a contradiction, and it is impossible to find commuting unitary dilations for $R, S, T$.

A. Lebow and R. Douglas have observed that a weaker example can be obtained more simply by taking the operator $A$ to be unitary. Also, the reader may have noticed that the dilation condition $P_{H} U_{R}^{k} U_{S}^{m} U_{T}^{n} \mid H=$ $R^{k} S^{m} T^{n}$ was not fully used. Actually, the counterexample is valid under the much weaker assumption that $P_{H} U_{S}\left|H=S, P_{H} U_{T}\right| H=T$, and $P_{H} U_{R}^{n} \mid H=R^{n}$ for $n \geqq 0$. Further, the assumption that $U_{T}$ be unitary was not used at all, and there are several ways that the example can be strengthened at the expense of minor complications. However, I do not know of any simple modification which will produce three proper contractions without commuting unitary dilations. 
A simple idea contained in the proof above sheds light on a recent result of Sz.-Nagy and Foias [9]. Let $R$ and $T$ be commuting contractions with $\|T\|=1$, acting on a Hilbert space $H$, and let $U_{R}, U_{T}$ be a pair of commuting unitary dilations acting on a Hilbert space $K$ containing $H$. (The existence of $U_{R}, U_{T}$ is guaranteed by Ando's theorem [1], [8, Chapter 1].) Let $K_{0}$ be the smallest subspace of $K$ which contains $H$ and reduces $U_{R}$, let $E$ be the projection on $K_{0}$, and let $\widetilde{T}=E U_{T} \mid K_{0}$. Then it is an elementary exercise to verify that $\widetilde{T}^{r}$ commutes with $U_{R} \mid K_{0}$ (the minimal unitary dilation for $R$ ), $\|\widetilde{T}\|=$ $\|T\|=1$, and the pair $U_{R} \mid K_{0}, \widetilde{T}$ dilates the pair $R, T$. The first two statements are trivial, and the last will be evident to anyone familiar with the structure of unitary dilations. For the reader's convenience a proof of the last statement is sketched in the next paragraph.

By definition, the space $K_{0}$ is the closed linear span of the spaces $U_{R}^{n} H,-\infty<n<\infty$. Let $K_{0}^{+}$be the closed linear span of the spaces. $U_{R}^{n} H, n \geqq 0$, and let $M=K_{0}^{+} \ominus H$. Then the following facts (a) and (b) are well-known and easy to verify:

(a) $K_{0} \ominus K_{0}^{+}$is the closed linear span of all vectors $\left(U_{R}^{* n}-R^{* n}\right) x$, with $x \in H$ and $n \geqq 0$

(b) $\quad M$ is the closed linear span of all vectors $\left(U_{R}^{n}-R^{n}\right) x$, with $x \in H$ and $n \geqq 0$.

From (a) and (b) we deduce:

(c) $\widetilde{T} K_{0}^{+} \subset K_{0}^{+}$. This follows from (a) and a routine computation showing that for all $x, y \in H$ and $m, n \geqq 0,\left(\widetilde{T} U_{R}^{m} x,\left(U_{R}^{* n}-R^{* n}\right) y\right)=$ $\left(E U_{T} U_{R}^{m} x,\left(U_{R}^{* n}-R^{* n}\right) y\right)=\left(E U_{T} U_{R}^{m+n} x, y\right)-\left(E U_{T} U_{R}^{m} x, R^{* n} y\right)=\left(T R^{m+n} x\right.$, $y)-\left(T R^{m} x, R^{* n} y\right)=0$.

(d) $\widetilde{T} M \subset M$. This follows from (b) and a similar computation showing that for all $x, y \in H$ and $m, n \geqq 0,\left(\widetilde{T}\left(U_{R}^{m}-R^{m}\right) x, U_{R}^{* n} y\right)=0$. Finally we compute, for $x, y \in H$ and $m, n>0$,

$$
\begin{aligned}
\left(\widetilde{T}^{m} U_{R}^{n} x, y\right) & =\left(\widetilde{T}^{m} R^{n} x, y\right)+\left(\widetilde{T}^{m}\left(U_{R}^{n}-R^{n}\right) x, y\right) \\
& =\left(\widetilde{T}^{m} R^{n} x, y\right)=\left(T^{m} R^{n} x, y\right) .
\end{aligned}
$$

The vanishing of $\left(T^{m}\left(U_{R}^{n}-R^{n}\right) x, y\right)$ follows from (b) and (d), and the last equality follows from (c) and (d) (see [7], Lemma 0, for a complete proof.)

The relation $\|\widetilde{T}\|=\|T\|$ depended on the assumption $\|T\|=1$, but if we replace $T$ by $T /\|T\|$ and apply similar reasoning, we obtain the following result, which is equivalent to the main result of [9] (see [3] for details).

Theorem (Sz.-Nagy and Foias). Let $R$ and $T$ be commuting contractions on a Hilbert space $H$, and let $U$ be the minimal unitary dilation of $R$ to a Hilbert space $K$. Then there exists an operator 
$\widetilde{T}$ on $K$ such that:

(i) The pair $U, \widetilde{T}$ dilates the pair $R, T$.

(ii) $U \widetilde{T}=\widetilde{T} U$

(iii) $\|\widetilde{T}\|=\|T\|$.

Therefore, the result of Sz.-Nagy and Foias may be viewed as a consequence of Ando's theorem on the existence of unitary dilations for a pair of commuting contractions. Conversely, it is easy to deduce Ando's theorem from the theorem above, and in effect, the two results are equivalent. However, the clever proof of Sz.-Nagy and Foias in [9] proceeds from first principles, and does not rely on Ando's theorem (as does the proof above.) Another proof, written in matricial notation and also independent of Ando's theorem, may be found in [3].

4. Proper contractions without commuting unitary dilations. In this section we give an example of three commuting proper contractions which fail to have commuting unitary dilations. Unfortunately, this example is not conveniently expressable in functional notation, and we are forced to use the more cumbersome sequential notation.

We begin as in the preceding example. Let $H_{0}$ be a Hilbert space of dimension at least two, and let $H=H_{0} \oplus H_{0}$. Choose noncommuting isometries $V_{2}$ and $V_{3}$ on $H_{0}$, and define operators $S_{1}, S_{2}, S_{3}$ on $H$ by the operator matrices:

$$
S_{i}=\left[\begin{array}{ll}
0 & 0 \\
V_{i} & 0
\end{array}\right]
$$

where $V_{1}$ is the identity operator on $H_{0}$. We shall show that the operators $\lambda S_{i}, i=1,2,3$, do not have commuting unitary dilations when $\lambda$ is sufficiently close to $1,0<\lambda<1$. The idea is that commuting unitary dilations for $\lambda S_{i}$ would have to converge, as $\lambda \rightarrow 1$, to commuting unitary dilations for $S_{i}$, and this would contradict the result of $\S 3$.

The minimal unitary dilation $U(\lambda)$ for $\lambda S_{1}, 0<\lambda<1$, may be realized as follows on the space $K_{0}$ of all sequences $\left\{\cdots,\left(x_{-2}, x_{-1}\right)\right.$, $\left.\overline{\left(x_{0}, x_{1}\right)},\left(x_{2}, x_{3}\right), \cdots\right\}$ of elements of $H=H_{0} \oplus H_{0}$. (The zero' the component is boxed, and the space $H$ is identified with the subspace of $K_{0}$ consisting of all sequences which vanish outside the box.)

$$
\begin{aligned}
& U(\lambda)\left\{\cdots,\left(x_{-2}, x_{-1}\right), \overline{\left(x_{0}, x_{1}\right)},\left(x_{2}, x_{3}\right), \cdots\right\} \\
&=\left\{\cdots,\left(x_{-4}, x_{-3}\right),\left(x_{-2}, \sqrt{1-\lambda^{2}} x_{-1}+\lambda x_{0}\right)\right. \\
&\left.\left(\sqrt{1-\lambda^{2}} x_{0}-\lambda x_{-1}, x_{1}\right),\left(x_{2}, x_{3}\right), \cdots\right\} .
\end{aligned}
$$


Note that $U(1)$ is a unitary dilation (but not the minimal unitary dilation) for $S_{1}$, and the operators $U(\lambda)$ converge uniformly to $U(1)$ as $\lambda \rightarrow 1$. Let $M$ denote the smallest subspace of $K_{0}$ which contains $H$ and reduces $U(1)$. Expressed concretely, $M$ is the space of all sequences

$$
\left\{\cdots,\left(x_{-4}, 0\right),\left(x_{-2}, 0\right),\left(x_{0}, x_{1}\right),\left(0, x_{3}\right),\left(0, x_{5}\right), \cdots\right\} .
$$

Now for each fixed $\lambda, 0<\lambda<1$, we assume the existence of commuting unitary dilations $U_{i}(\lambda)$ for $\lambda S_{i}, i=1,2,3$, and there is no loss of generality in assuming that for all $\lambda, 0<\lambda<1$, the operators $U_{i}(\lambda)$ act on a fixed Hilbert space $K$ containing $H$. If $K_{0}(\lambda)$ is the smallest subspace of $K$ which contains $H$ and is invariant under $U_{1}(\lambda)$, then by the uniqueness theorem of Sz.-Nagy quoted in $\$ 2$, we may assume that $K_{0}(\lambda)=K_{0}$ for all $0<\lambda<1$, and that $U_{1}(\lambda) \mid K_{0}$ is the operator $U(\lambda)$ defined above. (Given unitary operators $W(\lambda)$ which map $K_{0}(\lambda)$ onto $K_{0}$, fix all elements of $H$, and satisfy

$$
W(\lambda)\left(U_{1}(\lambda) \mid K_{0}(\lambda)\right) W(\lambda)^{-1}=U(\lambda),
$$

one can embed $K_{0}$ in a larger Hilbert space $K^{\prime}$ and choose arbitrary unitary operators $W^{\prime}(\lambda)$ mapping $K$ onto $K^{\prime}$ and satisfying $W^{\prime}(\lambda) \mid K_{0}(\lambda)=$ $W(\lambda)$. Then one can replace the operators $U_{i}(\lambda)$ by their unitary transforms $W^{\prime}(\lambda) U_{i}(\lambda) W^{\prime}(\lambda)^{-1}$. For fixed $\lambda$, these new operators are again commuting unitary dilations for $\lambda S_{i}, i=1,2,3$, and, by construction, $W^{\prime}(\lambda) U_{1}(\lambda) W^{\prime}(\lambda)^{-1} \mid K_{0}=U(\lambda)$.)

The unit ball of operators is compact in the weak operator topology, and hence there exists a sequence $\left\{\lambda_{n}\right\}, \lambda_{n} \rightarrow 1$, such that the operators $P_{M} U_{i}\left(\lambda_{n}\right) P_{M}$ converge weakly, as $n \rightarrow \infty$, to operators $Q_{i}, 1,2,3$. Since $U_{1}(\lambda) \mid K_{0}=U(\lambda)$, the uniform convergence of $U(\lambda)$ implies that $P_{M} U_{1}(\lambda) P_{M}$ converges uniformly to $Q_{1}$, and that $Q_{1}|M=U(1)| M$. Further, it is routine to verify that $P_{H} Q_{i} \mid H=S_{i}, i=1,2,3$.

Now we show that $Q_{1}$ commutes with $Q_{2}$ and $Q_{3}$. Writing $E=P_{M}$ and using the notation $[A, B]=A B-B A$, we first note:

$$
\left[Q_{1}, Q_{i}\right]=\text { weak } \lim _{n \rightarrow \infty}\left[E U_{1}\left(\lambda_{n}\right) E, E U_{i}\left(\lambda_{n}\right) E\right]
$$

$$
\begin{aligned}
& \lim _{n \rightarrow \infty}\left\|(1-E) U_{1}\left(\lambda_{n}\right) E\right\|=0 \\
& \lim _{n \rightarrow \infty}\left\|E U_{1}\left(\lambda_{n}\right)(1-E)\right\|=0 .
\end{aligned}
$$

Equation (1) holds because $E U_{1}\left(\lambda_{n}\right) E$ converses uniformly to $Q_{1}$, and (2) and (3) follow immediately from the expression for $U(\lambda)$ above. Now the identity

$$
\begin{aligned}
{\left[E U_{1}(\lambda) E, E U_{i}(\lambda) E\right]=} & E\left[U_{1}(\lambda), U_{i}(\lambda)\right] E+E U_{i}(\lambda)\left\{(1-E) U_{1}(\lambda) E\right\} \\
& -\left\{E U_{1}(\lambda)(1-E)\right\} U_{i}(\lambda) E
\end{aligned}
$$


together with (1), (2), (3), and $\left[U_{1}(\lambda), U_{i}(\lambda)\right]=0$ imply that $\left[Q_{1}, Q_{i}\right]=0$.

The contractions $Q_{i}$ leave $M$ invariant, $Q_{i} \mid M$ commutes with $Q_{1}|M=U(1)| M$, and $P_{H} Q_{i} \mid H=S_{i}, 1,2,3$. From this we conclude (the argument is identical to one in $\S 3$ ) that the action of $Q_{i}$ on $M$ is given by:

$$
\begin{aligned}
& Q_{i}\left\{\cdots,\left(x_{-2}, 0\right), \overline{\left(x_{0}, x_{1}\right)},\left(0, x_{3}\right), \cdots\right\} \\
& =\left\{\cdots,\left(V_{i} x_{-4}, 0\right), \overline{\left(V_{i} x_{-2}, V_{i} x_{0}\right)},\left(0, V_{i} x_{1}\right), \cdots\right\} .
\end{aligned}
$$

Of course, $Q_{2}$ and $Q_{3}$ do not commute because $V_{2}$ and $V_{3}$ do not commute. However, we will now show that the method of construction of $Q_{2}$ and $Q_{3}$ implies that they must commute. This is a contradiction, and therefore, the commuting unitary dilations $U_{i}(\lambda)$ cannot exist when $\lambda$ is sufficiently close to $1,0<\lambda<1$.

To see that $Q_{2}$ and $Q_{3}$ must commute, first note that their representation above shows that each is isometric on $M$. As is wellknown, a sequence of contractions which converges weakly to an isometry also converges in the strong operator topology. Since the isometry $Q_{i} \mid M$ is a weak (hence strong) limit of the contractions $P_{M} U_{i}\left(\lambda_{n}\right) P_{M} \mid M$, elementary properties of the strong operator topology imply that for all $x$ in $M$,

(a) $Q_{2} Q_{3} x=\lim _{n \rightarrow \infty} P_{M} U_{2}\left(\lambda_{n}\right) P_{M} U_{3}\left(\lambda_{n}\right) x$

(b) $\lim \left(1-P_{M}\right) U_{i}\left(\lambda_{n}\right) x=0$.

From this we obtain, for all $x$ in $M$,

$$
\begin{aligned}
& Q_{2} Q_{3} x=\lim P_{M} U_{2}\left(\lambda_{n}\right) P_{M} U_{3}\left(\lambda_{n}\right) x \\
& \quad=\lim P_{M} U_{2}\left(\lambda_{n}\right) U_{3}\left(\lambda_{n}\right) x+\lim P_{M} U_{2}\left(\lambda_{n}\right)\left(P_{M}-1\right) U_{3}\left(\lambda_{n}\right) x \\
& \quad=\lim P_{M} U_{2}\left(\lambda_{n}\right) U_{3}\left(\lambda_{n}\right) x=\lim P_{M} U_{3}\left(\lambda_{n}\right) U_{2}\left(\lambda_{n}\right) x=Q_{3} Q_{2} x .
\end{aligned}
$$

The proof is complete.

5. Remarks on an open problem. It has long been known that questions involving unitary dilations are often closely related to questions involving spectral sets. For instance, Von Neumann's theorem stating that the unit disc is a spectral set for every contraction is a simple consequence of the existence of a unitary dilation for a single contraction together with the easy fact that the disc is a spectral set for any unitary operator. Conversely, if a simply connected subset of the plane is a spectral set for an operator, then the operator has a normal dilation with spectrum in the boundary of the set $[2,4,5]$. (When the set in question is the unit disc, this says that Von Neumann's theorem implies that every contraction has a unitary dilation.) 
One can try to generalize Von Neumann's theorem to apply to several commuting contractions as follows. Let $D$ be the unit disc $\{z|| z \mid \leqq 1\}$ in the complex plane, and let $D^{n}$ denote the $n$-fold direct product of $D$ with itself. We shall say that $D^{n}$ is a spectral set for $n$ commuting contractions $S_{1}, \cdots, S_{n}$ if for each polynomial $p\left(z_{1}, \cdots, z_{n}\right)$ in $n$ variables,

$$
\left\|p\left(S_{1}, \cdots, S_{n}\right)\right\| \leqq\|p\| \infty,
$$

where $\|p\| \infty$ denotes the maximum of $\left|p\left(z_{1}, \cdots, z_{n}\right)\right|$ on $D^{n}$. The following conjecture is a natural generalization of Von Neumann's theorem.

Conjecture. The $n$-polydisc $D^{n}$ is a spectral set for any $n$ commuting contractions on a Hilbert space.

It is easy to employ the spectral theorem to show that this conjecture is true if the commuting contractions are normal operators. From this, it follows that the existence of commuting unitary dilations for $n$ commuting contractions implies the conjecture for that $n$. Thus Ando's theorem implies that the conjecture is true for $n=2$. For $n \geqq 3$, the conjecture is still open, and the results of this paper show that its proof (if, indeed, it is true) cannot rely on the existence of commuting unitary dilations for commuting contractions.

Also, the operators of $\S 3$ cannot be used to construct a counterexample to the conjecture, for $D^{3}$ is a spectral set for any three contractions defined on $H=H_{0} \oplus H_{0}$ by operator matrices

$$
S_{i}=\left[\begin{array}{ll}
0 & 0 \\
Q_{i} & 0
\end{array}\right], i=1,2,3,\left\|Q_{i}\right\| \leqq 1
$$

This also shows that the spectral set problem and the commuting unitary dilation problem are not equivalent for $n \geqq 3$.

To see that $D^{3}$ is a spectral set for the operators $S_{1}, S_{2}, S_{3}$, consider an arbitrary polynomial $p$ in three variables:

$$
p\left(z_{1}, z_{2}, z_{3}\right)=a_{0}+\sum_{i=1}^{3} a_{i} z_{i}+q\left(z_{1}, z_{2}, z_{3}\right),
$$

where $q$ is a polynomial containing only terms of second or higher degree. Then

$$
p\left(S_{1}, S_{2}, S_{3}\right)=\left[\begin{array}{ll}
a_{0} & 0 \\
\sum_{i=1}^{3} a_{i} Q_{i} & a_{0}
\end{array}\right] .
$$

The first step, left to the reader, is to verify that 


$$
\left\|\left[\begin{array}{ll}
a_{0} & 0 \\
\sum_{i=1}^{3} a_{i} Q_{i} & a_{0}
\end{array}\right]\right\| \leqq\left\|\left[\begin{array}{ll}
\left|a_{0}\right| & 0 \\
\sum_{i=1}^{3}\left|a_{i}\right| & \left|a_{0}\right|
\end{array}\right]\right\| .
$$

Hence to show that $D^{3}$ is a spectral set for $S_{1}, S_{2}, S_{3}$, we need only show that the norm of the latter matrix is no larger than

$$
\inf \left\|a_{0}+\sum_{i=1}^{3} a_{i} z_{i}+q\left(z_{1}, z_{2}, z_{3}\right)\right\| \infty,
$$

where the infemum is taken over all polynomials $q$ containing only terms of degree two or higher.

Now a classical result of Caratheodory and Fejer states that

$$
\inf \left\|b_{0}+b_{1} z+r(z)\right\| \infty=\left\|\left[\begin{array}{cc}
b_{0} & 0 \\
b_{1} & b_{0}
\end{array}\right]\right\|
$$

where the infemum is taken over all polynomials $r(z)$ (in one variable) which contain only terms of degree two or higher. (For a modern proof, see Sarason's beautiful paper [6], where the result is derived as a consequence of a special case of the theorem of Sz.-Nagy and Foias discussed in §3.) Using this fact, we have:

$$
\begin{aligned}
\left\|p\left(S_{1}, S_{2}, S_{3}\right)\right\| & \leqq\left\|\left[\begin{array}{cc}
\left|a_{0}\right| & 0 \\
\sum_{i=1}^{3}\left|a_{i}\right| & \left|a_{0}\right|
\end{array}\right]\right\| \\
& =\inf \left\|\left|a_{0}\right|+\left(\sum_{i=1}^{3}\left|a_{i}\right|\right) z+r(z)\right\| \infty \\
& \leqq \inf \left\|\left|a_{0}\right|+\sum_{i=1}^{3}\left|a_{i}\right| z_{i}+q\left(z_{1}, z_{2}, z_{3}\right)\right\| \infty \\
& =\inf \left\|a_{0}+\sum_{i=1}^{3} a_{i} z_{i}+q\left(z_{1}, z_{2}, z_{3}\right)\right\|_{\infty},
\end{aligned}
$$

where, again, $r$ and $q$ range over all polynomials, in one and three variables, respectively, containing only terms of degree two or higher. The second inequality was obtained by setting all three variables equal in the polynomial $\left|a_{0}\right|+\sum_{i=1}^{3}\left|a_{i}\right| z_{i}+q\left(z_{1}, z_{2}, z_{3}\right)$, and the last equality was obtained by multiplying by $a_{0} /\left|a_{0}\right|$ and replacing $z_{i}$ by $\left(\bar{a}_{0} /\left|a_{0}\right|\right) \cdot\left(a_{i} /\left|a_{i}\right|\right) z_{i} \cdot$

Thus $D^{3}$ is a spectral set for three commuting contractions which admit no commuting unitary dilations, and it appears that the connection between spectral sets and unitary dilations may not be as close as has been assumed. 


\section{REFERENCES}

1. T. Ando, On a pair of commutative contractions, Acta. Sci. Math. 24 (1963), 88-90.

2. C. A. Berger, Normal dilations, Doctoral Dissertation, Cornell University, 1963.

3. R. G. Douglas, P.S. Muhly, and C. Pearcy, Lifting commuting operators, Michigan Math. J. 15 (1968), 385-395.

4. C. Foias, Some applications of spectral sets I, Acad. R. P. Romine, Stud. Cerc. Math. 10 (1959), 365-401 (in Romanian).

5. A. Lebow, On von Neumann's theory of spectral sets, J. Math. Anal. (1963), 64-90.

6. D. Sarason, Generalized interpolation in $H^{\infty}$, Trans. Amer. Math. Soc. 127 (1967), 179-203.

7. - On spectral sets having connected complement, Acta Sci, Math. (Szeged) 26 (1965), 289-299.

8. B. Sz.-Nagy and C. Foias, Analyse harmonique des operaturs de l'espace de Hilbert, Akademai Kiado Budapest, 1967.

9. —_ Compte Rendus Acad. Sc. Paris 266 (1968), 493-495.

Received June 9, 1969, and in revised form January 5, 1970.

University of Massachusetts at Boston 


\title{
PACIFIC JOURNAL OF MATHEMATICS
}

\author{
EDITORS
}

\author{
H. SAMELSON \\ Stanford University \\ Stanford, California 94305 \\ RichaRd PIERCE \\ University of Washington \\ Seattle, Washington 98105
}

J. DugundJI

Department of Mathematics

University of Southern California

Los Angeles, California 9.0007

RICHARD ARENS

University of California

Los Angeles, California 9.0024

\section{ASSOCIATE EDITORS}

E. F. BeCKenBACH
B. H. NeumanN

F. WoLE

K. YoSHIDA

\section{SUPPORTING INSTITUTIONS}

\author{
UNIVERSITY OF BRITISH COLUMBIA \\ CALIFORNIA INSTITUTE OF TECHNOLOGY \\ UNIVERSITY OF CALIFORNIA \\ MONTANA STATE UNIVERSITY \\ UNIVERSITY OF NEVADA \\ NEW MEXICO STATE UNIVERSITY \\ OREGON STATE UNIVERSITY \\ UNIVERSITY OF OREGON \\ OSAKA UNIVERSITY \\ UNIVERSITY OF SOUTHERN CALIFORNIA
}

\author{
STANFORD UNIVERSITY \\ UNIVERSITY OF TOKYO \\ UNIVERSITY OF UTAH \\ WASHINGTON STATE UNIVERSITY \\ UNIVERSITY OF WASHINGTON \\ AMERICAN MATHEMATICAL SOCIETY \\ CHEVRON RESEARCH CORPORATION \\ TRW SYSTEMS \\ NAVAL WEAPONS CENTER
}

The Supporting Institutions listed above contribute to the cost of publication of this Journal, but they are not owners or publishers and have no responsibility for its content or policies.

Mathematical papers intended for publication in the Pacific Journal of Mathematics should be in typed form or offset-reproduced, (not dittoed), double spaced with large margins. Underline Greek letters in red, German in green, and script in blue. The first paragraph or two must be capable of being used separately as a synopsis of the entire paper. The editorial "we" must not be used in the synopsis, and items of the bibliography should not be cited there unless absolutely necessary, in which case they must be identified by author and Journal, rather than by item number. Manuscripts, in duplicate if possible, may be sent to any one of the four editors. Please classify according to the scheme of Math. Rev. Index to Vol. 39. All other communications to the editors should be addressed to the managing editor, Richard Arens, University of California, Los Angeles, California, 90024.

50 reprints are provided free for each article; additional copies may be obtained at cost in multiples of 50 .

The Pacific Journal of Mathematics is published monthly. Effective with Volume 16 the price per volume (3 numbers) is $\$ 8.00$; single issues, $\$ 3.00$. Special price for current issues to individual faculty members of supporting institutions and to individual members of the American Mathematical Society: $\$ 4.00$ per volume; single issues $\$ 1.50$. Back numbers are available.

Subscriptions, orders for back numbers, and changes of address should be sent to Pacific Journal of Mathematics, 103 Highland Boulevard, Berkeley, California, 94708.

PUBLISHED BY PACIFIC JOURNAL OF MATHEMATICS, A NON-PROFIT CORPORATION

Printed at Kokusai Bunken Insatsusha (International Academic Printing Co., Ltd.), 7-17, Fujimi 2-chome, Chiyoda-ku, Tokyo, Japan. 


\section{Pacific Journal of Mathematics}

\section{Vol. 34, No. $2 \quad$ June, 1970}

Shair Ahmad, On the oscillation of solutions of a class of linear fourth order

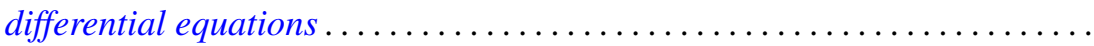

Leonard Asimow and Alan John Ellis, Facial decomposition of linearly

compact simplexes and separation of functions on cones ..............

Kirby Alan Baker and Albert Robert Stralka, Compact, distributive lattices of

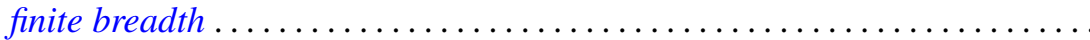

James W. Cannon, Sets which can be missed by side approximations to

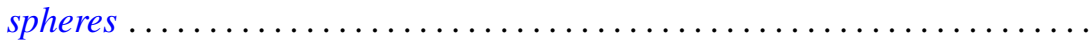

Prem Chandra, Absolute summability by Riesz means .................. 335

Francis T. Christoph, Free topological semigroups and embedding topological semigroups in topological groups....

Henry Bruce Cohen and Francis E. Sullivan, Projecting onto cycles in smooth, reflexive Banach spaces.................................

John Dauns, Power series semigroup rings .......................

Robert E. Dressler, A density which counts multiplicity ................

Kent Ralph Fuller, Primary rings and double centralizers ................

Gary Allen Gislason, On the existence question for a family of products.......

Alan Stuart Gleit, On the structure topology of simplex spaces .............

William R. Gordon and Marvin David Marcus, An analysis of equality in

certain matrix inequalities. $I \ldots \ldots \ldots \ldots \ldots \ldots \ldots \ldots$

Gerald William Johnson and David Lee Skoug, Operator-valued Feynman

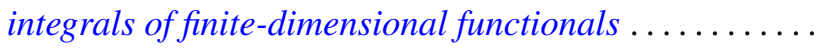

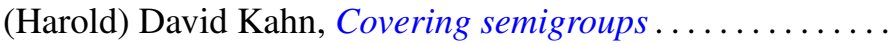

Keith Milo Kendig, Fibrations of analytic varieties

Norman Yeomans Luther, Weak denseness of nonatomic measures on perfect,

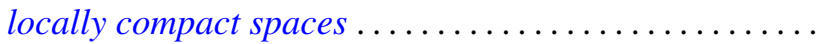

Guillermo Owen, The four-person constant-sum games; Discriminatory solutions on the main diagonal ...

Stephen Parrott, Unitary dilations for commuting contractions

Roy Martin Rakestraw, Extremal elements of the convex cone $A_{n}$ of

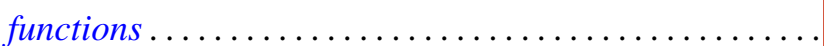

Peter Lewis Renz, Intersection representations of graphs by

William Henry Ruckle, Representation and series summability of complete

biorthogonal sequences.

F. Dennis Sentilles, The strict topology on bounded sets ...

Saharon Shelah, A note on Hanf numbers ...

Harold Simmons, The solution of a decision problem for several classes of rings. . .

Kenneth S. Williams, Finite transformation formulae involving the Legendre 